\title{
A NEW THEORY OF HEARING.
}

\author{
Review. \\ A New Theory of Cochlear Mechanism: An Enquiry into the Analytical \\ Mechanism of the Internal Ear. By Sir. Thomas Wrightson and \\ Arthur Keith. London: Macmillan \& Co., Ltd., 1918. 8vo., \\ pp. 254. Price 12s. 6d. net.
}

In this most interesting book Sir Thomas Wrightson and Prof. Arthur Keith give an explanation of the mechanism of heariuir which differs from previous theories in several important respects.

Waves of sound travelling along the external auditory meatus are received on the drum membrane and conveyed by the chain of ossicles to the footplate of the stapes. The labyrinth is regarded as a closed cavity, whose walls are rigid except at the fenestra ovalis and the fenestra rotunda. This cavity is filled with incompressible fluid consisting of perilymph and endolymph. Any impulse imparted to this fuid is transmitted instantaneously thronghout its whole mass. The scala vestibuli and scala media are, for practical purposes, one cavity, the two being separated only by the thin membrave of Reissner, which merely prevents the two fluids from mixing. Any sudden increase of pressure caused by the movement inwards of the footplate of the stapes causes a displacement of the basilar membrane towards the scala tympani and a corresponding outward movement of the membrane of the fenestra rotunda. As the stapes moves outwards again the basilar membrane rises and the feuestra rotunda recedes. This up-and-down movement of the basilar membrane takes place along its whole length simultaneously. Very little, if any, of the fluid of the scala vestibuli actually passes the helicotrema, as its impulse has already been transmitted to the scala tympani by the displacement of the basilar membrane. The up-and-down movement of the basilar membrane causes a motion from side to side of the organ of Corti, the hairs of which are embedded in the membrana tectoria. This latter structure does not move from side to side, and the result is that the hairs are bent with the varying phases of the wave, and the nerveendings thus stimulated send periodic impulses to the brain, where they are analysed into the sensation of sound.

Wrightson commences the exposition of his theory with a dissertation on wave forms and wave motion. There are several elementary facts which it is necessary to bring before the reader.

In a simple wave the crests and hollows are equidistant and periodic. In compound waves this is no longer the case. In the study of compound wave forms Wrightson demonstrated many years ago that "although the distances apart of crests and hollows were no longer equal, ret if the crossing-points of the compound curve on the axis were regarded as impulse points as well as the maxima and minima of the crests and hollows, there were revealed to the eye nunerous systems of consecutive impulses which not only satisfied the requirements of individual frequency for the simple tones, but also systems which were necessary to produce the differential and summational tones." He shows that in a simple wave there is a change of momentum at the crossing-points. In a simple sound-wave there are four distinct phases in each complete cycle, each of which produces a separate impulse on the nerve-endings of the organ of hearing.

Sound-waves of condensation and rarefaction strike the drum mem- 
brane and set it in motion. The motion of the drum membrane, into which is fixed the handle of the malleus, is conveyed through the incus to the stapes. The malleus and incus together form a bent lever, the long end of which is represented by the handle of the malleus, and the short end by the long process of the incus. The hinge of this lever is roughly along a line between the anterior process of the malleus and the posterior end of the incus. The leverage has been calculated as a ratio of 3 to 1. The area of the drum membrane is about twenty times that of the footplate of the stapes, and this would give a total increase of effective pressure of $20 \times 3$ or 60 to 1 , which is transmitted by the stapes to the fluid in the labyrinth. It has been shown that the stapes has not a simple piston action, but rather that of a lever with the hinge near the posterior end of the footplate. The margin of the footplate is fixed in the fenestra ovalis by a strong annular ligament. The fibres of this annular ligament are much shorter at the posterior end than at the anterior end. This allows of only very limited movement at the posterior end, while the anterior end can move much more freely.

Connected with the chain of ossicles are two muscles-the tensor tympani and the stapedius. The tensor tympani is attached to the malleus, and its action is to pull the drum inwards. The stapedius extends forwards from the pyramid to be attached to the head of the stapes. Its action is to pull the stapes slightly out of the oval window. These two muscles then act in opposition to each other, and constitute an elastic balance which tends to bring the ossicles back into a position of rest.

The labyrinth is regarded by Wrightson as a closed box with a piston, represented by the stapes at one end and at the other end by the nembrane of the round window. Any increase of pressure caused by the inward movement of the stapes is transmitted instantaneously throughout all the fluid contents of the labyrinth, and causes a simultaneous outward movement of the membrane of the round window, and vice versa. The cavity of the cochlea is divided into two main cavities by the bony spiral lamina and the basilar membrane. The upper passage, or scala ve'stibuli, contains the endolymphatic scala media, which is only separated from the perilymph by the thin Reissuer's membrane.

The basilar membrane is narrowest at the commencement in the basal coil and broadest at the helicotremal end. In the basal coil it is $0.17 \mathrm{~mm}$. in breadth and at the apex $0.400 \mathrm{~mm}$. It is thus between two and three times as broad in the apical coil as in the basal coil. The organ of Corti is placed on the basilar membrane at its junction with the spiral lamina. It contains two sets of hair-cells, the hairs of which are embedded in the soft membrana tectoria. The hair-cells are supported by the cells of Deiters and the arch-shaped rods of Corti. One of the feet of the arch rests on the basilar membrane at its junction with the spiral lamina, the other foot rests on the basilar membrane a short distance further out. The basilar membrane is composed for the most part of strong transverse fibres which are relatively unyielding, but that part which is immediately under the arch of Corti is of a different structure from the free portion, as the fibres are here absent and the membrane is thinner and more yielding.

When the stapes moves inwards the displacement of the fluid is along the scala vestibuli in the direction of the round window at the foot of the scala tympani. Now "the relative areas of the helicotrema and the basilar membrane are respectively as 1 to 81 , according to Dr. Keith's measurements, so that the liquid displacements will naturally select the 
shortest route and the line of least resistance to the fenestra yotunda, thus passing by way of the basilar membrane in preference to the helicotrema, the object of which aperture seems principally to be to form a liquid continuity between the vestibular and tympanic passage, securing equality in the distribution of fluid in these two passages." "The object of the reduction of area in the vestibular and tympanic passages, apart from the spreading out of the influence of the motion to an extended line of nerves, to which I have already alluded, ppears to be to reduce the mass of liquid moved."

" Between the vestibular and trmpanie passages we have two flexible membranes, the basilar and the Reissnerian forming two sides of the triangular-shaped scala media (cochlear canal), the liquid endolymph filling this space. These two membranes pass the displacements from one scala to the other, though no particles of fluid pass through either of the membranes. Thus the displacements of the fenestra ovalis, Reissnerian, and basilar membranes, and the fenestra rotunda are equal, and the internal membranes move as though they form part of the liquid."

The estimated area of the basilar membrane is $13.3 \mathrm{sq} . \mathrm{mm}$., or nine times the effective area of the footplate of the stapes. The average area of the helicotrema is $0.175 \mathrm{sq}$. mm., or one-ninth of the effective area of the stapedial footplate. The ratios of the areas are therefore as follows :

$$
\begin{array}{ccc}
\text { Helicotrema. Fenestra ovalis. Basilar membrane. } \\
1
\end{array}
$$

" It is evident that, taking into consideration the relative areas of the basilar membrane and the helicotrema, the line of motion of the displacements will follow that of least resistance or through the basilar membrane, especially in the case of such rapid displacements as we have in soundwaves." Probably little or none of the fluid displacement in the scala vestibuli passes the helicotrema. All parts of the basilar membrane rise and fall to the same extent together. In each segment of the membrane the greatest excursion takes place, not at the centre, but near the junction of the fibrillary part with the subarcuate part. The more elastic subarcuate part stretches more than the outer fibrillary part when pressure is applied to it. This movement of the basilar membrane causes a sileto-side movement of the top of the arch of Corti, and therefore of the upper surface of the organ from which project the sensory hairs. The membrana tectoria does not participate in this side-to-side movement, and hence the hairs of the organ, which are embedded in it, are subjected to a bending from side to side.

Each movement inwards of the footplate of the stapes causes a depression of the basilar membrane, a movement of the Corti arch away from the lamina spiralis and the modiolus, and a bending of the hairs towards the modiolus. When the stapes moves outwards the hairs are bent away from the lamina.

Wrightson assumes that each change of momentum of the fluid, however small, produces a stimulus. He shows how each hair-cell receives four stimuli in each complete cycle of a simple sound-wave. In the first phase, or inward movement of the stapes, the hairs are bent towards the modiolus. In the second phase the stapes is brought back to a state of equilibrium by the elastic resistance of the muscles, and the hair returns from its bent position to the upright. In the third phase the stapes moves outwards and the hair is bent away from the modiolus. In the fourth phase the hair returns to its normal upright position.

In the case of compound sound-waves every modification of the curve 
produces a change of position of the hairs, and thus a series of periodic impulses are sent to the brain in exactly the order and time-relationship in which they were received. They are finally analysed in the cortex of the brain.

There are two postulates upon which Wrightson's theory essentially depends, viz., firstly, that the labyrinth is a closed box containing incompressible liquid, the only outlet for increase in pressure of which is by the round window; secondly, that in a sound-wave not only the crests and hollows, but also the points where the curve crosses the axial line, are points of stimulation.

With regard to the first point, Wrightson and Keith ignore the presence of the endolymphatic and perilymphatic aqueducts. This omission may be intentional, but one cannot consider the theory as proved until it is shown that they have no influence on the movements of the fluids in the labyrinth. It is possible that the movements of the fluids in the labyrinth are so small and of such rapidity that the presence of the two aqueducts is immaterial, but we consider that this should have been made clear in the explanation of the theory. The diameter of the aqueduct of the cochlea at its narrowest point is not so very inconsiderable in comparison with the diameter of the scala tympani that its presence should be ignored. The ductus reuniens is certainly very minute and its walls appear to be normally in apposition, but the ductus endolymphaticus, with which it communicates iudirectly, is relatively wide, and has a large flattened saccular end under the dura of the posterior fossa of the skull. This sac, under the influence of increased pressure in the labyrinth, could expand into a more globular shape and allow of a displacement of endolymph relatively large in comparison with the total capacity of the labyrinth, and enormous in comparison with the capacity of the endolymph system. Again, the aqueduct of the cochlea could allow of a large displacement of perilymph from the scala tympani into the subarachnoid space, the voluue of which can by no means be regarded as constant. The cranial contents are so richly supplied with large, thinwalled blood-vessels that any increase or decrease of the total volume of the fluid in the cranium is at once compensated by the contraction or dilatation of these vessels. It is common knowledge that several cubic centimetres of cerebrospinal fluid (a quantity many times the total capacity of both labyrinths) may be drawn off without causing any appreciable disturbance to the subject.

Certainly the scala vestibuli, in which the movements of the labyrinthine fluids commence, has no outlet other than the helicotrema and the movement of the basilar membrane, but to Sir Thomas Wrightson the problem of working out what, if any, influence these aqueducts have on the movements of the labyrinthine fluid should not present any great difficulty.

With regard to the second essential to the theory, namely, that there are four stimuli in every complete cycle of a simple sound-wave, Wrightson places his data and deductions so convincingly before the reader that one must accept them as correct.

Wrightson explains the gradual widening of the basilar membrane towards the apex together with the narrowing of the cochlea as a means for distributing the stapedial displacements equally over the basilar membrane.

In this theory also the idea that the hair-cells of the organ of Corti are stimulated by a movement of flexion of the hairs brings them into line with the hair-cells of the ampullæ of the semicircular canals and 
otolith membranes, which we know to be stimulated by flexion of the hairs.

There are several facts which are difficult to reconcile with the displacement theory. Siebenmann's experiments showed that prolonged exposure of animals to the blast of a high-pitched whistle was followed by degeneration of the organ of Corti of the basal coil, a medium-toned whistle by degeneration in the middle coil, and a low-toned whistle of the apical coil. This appears to show that certain sounds are perceived by certain special parts of the basilar membrane. Keith, in his appendix, states - "This distributional mechanism of the cochlea will tend to confine displacements caused by vibrations of small amplitude to the basal part of the basilar membrane, while only waves of the greatest amplitude will reach the apical region." Amplitude of vibration is not the same thing as rapidity of vibration, and, in any case, the displacement theory does not allow of any selective action on any one part of the cochlea, as the whole basilar membrane would be equally affected by any one tone.

The explanation of bone-conduction is similarly unsatisfactory, and can be explained only in the case of the stapes being fixed, but not otherwise. This, however, is no great drawback to the theory, as none of the explanations of the various tuning-fork tests are really satisfactorily explained by the resonance theory either.

Whether the displacement theory as expounded by Wrightson and Keith be accepted or not, the anatomical researches of Keith render the resonance theory of Helmholtz untenable. It is impossible in an abstract such as this to go into the details of the question. Only the salient features are here given, and readers are referred to the original for further information.

Wrightson and Keith in this book have opened up new ground, and increased our knowledge of the mechanism of hearing in a way that has not been done since Helmholtz advanced his famous resonance theory many years ago. It is essential for all otologists and physiologists to study this book carefully if they wish to keep abreast of the times in their knowledge of the physiology of hearing. J.K. Milne Dickie.

\section{NOTES AND QUERIES.}

\section{A Case for Diagnosis.}

Dr. Neil Maclay (Newcastle) writes :

"Can any of your readers suggest an explanation of the following case?

"A male, aged thirty-two, with difficulty in swallowing; liquids go down pretty well, but solids hardly at all. No gullet growth, stricture, or spasm, and $\mathrm{X}$-ray of chest negative.

"The epiglottis is seen to lie in close contact with the posterior wall, and the bolus appears to sit upon its lingual surface. The left cord is paretic and nearly motionless, and the right cord is weak in abduction. There is no anæsthesia or paresis of the palate, but there is some weakness in the left sterno-mastoid and trapezius. No evidence of any other nerve involvement." 